\title{
An Information Geometry Approach for Unifying Mean Field Theories of Asymmetric Kinetic Ising Systems ${ }^{\dagger}$
}

\author{
Miguel Aguilera ${ }^{1,2}$, S. Amin Moosavi ${ }^{3}$ and Hideaki Shimazaki ${ }^{3}$ \\ 1 IAS-Research Center for Life, Mind, and Society, University of the Basque Country, Donostia, Spain \\ 2 ISAAC Lab, Aragón Institute of Engineering Research, University of Zaragoza, Zaragoza, Spain \\ 3 Graduate School of Informatics, Kyoto University, Kyoto, Japan \\ + Presented at the Entropy 2021: The Scientific Tool of the 21st Century, 5-7 May 2021; Available online: \\ https://sciforum.net/conference/Entropy2021/.
}

Published: 5 May 2021

Many physical and biological dynamical systems operate away from thermodynamic equilibrium, driven by their own activity as well as their interaction with the environment. The kinetic Ising model is a prototypical model for studying such non-equilibrium dynamics. Since its behaviour is generally intractable for large sizes due to combinatorial explosion, mean field theories are often employed to approximate network dynamics. However, mean field methods are often unable to capture systems displaying long-range correlations such as those operating near critical phase transitions. To tackle this problem, different variants of mean field approximations have been proposed for kinetic Ising models, each making unique assumptions about the correlation structure of the system. This disparity complicates the challenge of systematically advancing beyond previous contributions. Here, using information geometry, we propose that existing methods can be described and extended in a unified framework. Our method is defined as a family of expansions (called Plefka expansions) of an intractable marginal probability distribution around a specific point of a simplified model, defined in an information geometric space. These points are obtained by an orthogonal projection to a sub-manifold of probability distributions displaying a simplified correlation structure. This approach not only unifies previous methods but allows us to define novel methods that make unusual assumptions for mean field methods, like models preserving specific correlations of the system. By comparing analytic approximations and exact numerical simulations in a kinetic Sherrington Kirkpatrick model, we show that the new approximations found by our method provide more accurate estimates of the dynamics of the systems than classical equations, even near critical phase transitions presenting large fluctuations. In sum, our framework unifies and extends existing mean field methods in the kinetic Ising model from an information theoretic view, constituting a powerful tool for studying the dynamics of complex systems.

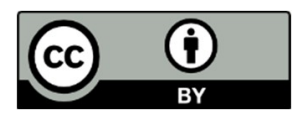

(C) 2021 by the authors. Licensee MDPI, Basel, Switzerland. This article is an open access article distributed under the terms and conditions of the Creative Commons Attribution (CC BY) license (http://creativecommons.org/licenses/by/4.0/). 